\title{
The Development of Power Transformers Diagnosis Framework Using Infrared Thermograms Analysis
}

\author{
Phoutsavanh Chandavong and Danu Wiroteurairuang
}

\begin{abstract}
A power transformer is very important and considered to be the most expensive equipment of the substation. Major problems that may occur to power transformers are found in bushing heat, radiator, on-load tap change and Tank. The reason for this problem is due to a lot of working load and a long operation time of transformers. It will make these points damage and expose to high temperature. If it has been left for a long time, any catastrophe would happen in the future. To detect anomalies and predict possible faults or check the quality of transformers, this paper proposes the development mechanism framework to monitor the temperature variations, and analyze hot regions of transformers using infrared thermograms. This method is economy, convenient and efficient.
\end{abstract}

Index Term-Infrared thermogram, power transformer, image processing.

\section{INTRODUCTION}

Nowadays, Electricity Du Lao (EDL) is responsible for the continuation activities in construction of power stations, transmissions, and power distributions, in order to meet the increasing needs for electricity. After the completion and operation of such facilities, maintenances are required for the next step to preserve the integrity, and safety of the power system. The maintenances of power system or equipment could be varied. This research represents the method of power equipment diagnosis using thermograms analysis. Thermal camera could be used for scanning the equipment surfaces without direct-contact to avoid surface damages. To rapidly detect problems or deterioration that could happen unexpectedly, the capability in reducing time used for solving problems and maintenance is needed according to the following issues:

Internal and external defection of equipment: for example, loose joint, loose contact, overload, unbalanced load, and improper installation. These defections usually cause excess heat, which could be harmful to equipment. Equipment failure needs high maintenance cost and maintenance staff which whom are risky for any accidents or life threatening. Temperature is a vital factor in status evaluation of electrical equipment. Therefore, temperature monitoring is considered to be one of the best maintenance methods.

Infrared thermogram (IRT) can be used for remote temperature measurement, which resulted by various temperature distribution on equipment surface shown in the thermogram image. It helped reducing maintenance cost, uncontrollable functions of the system, or system failure, encouraging appropriate maintenance staff planning, and

Manuscript received December 29, 2017; revised March 10, 2018

The authors are with Ubon Ratchathani Rajabhat University,Thailand (e-mail: Chandavong9@gmail.com). extending durability of the equipment.

Infrared thermogram technology is flourishing and being acceptable to be used prevalently as a tool for status monitoring in energy industries. This research encompasses thermogram analysis in diagnosis of the most important equipment in power station:-the transformer. Specific parts of transformers that needed to be focused are 4 components including tank, on-load \& off-load tap changer (OLTC), radiator, and bushing.

Notice that thermogram has been applied for probability analysis of possible damages by using temperature distribution patterns that could affect transformer defects. Z. A. Jaffery and A. K. Dubey applied the environmental friendly method in temperature monitoring and analysis of heat in electrical equipment by gradually changing temperature and analyzing the heat transfer within the hot region of the equipment using infrared themography. This could help protecting electrical equipment from further catastrophe in the future by defective prediction [1].

M. S. Jadin and S. Taib emphasized the benefits of infrared thermogram by using it for equipment diagnosis, which became a very effective for electrical equipment maintenance according to the performance speed and accuracy [2]. Y. C. Chou and L. Yao initialized the automatic diagnostic system for examining electrical equipment defects using infrared thermography [3].

The increasing temperature can cause equipment failure. Accordingly, heat detection pays an important role in identifying the initial problem. Equipment failure detection system using thermography is economy, reliable, and being used widely in order to determine and diagnose the equipment [4]. Y. C. Chou and L. Yao also papplied HSI color scheme for thermogram processing to obtain equipment failure detection and also proposed an automatic equipment diagnostic based on protection-oriented methodology in order to detect problems without damaging the equipment. To examine the equipment without damaging it, infrared thermography could be the best choice comparing to the others.

In this paper, thermogram processing using statistics method and cluster processing can help in identifying Region Of Interest (ROI) and relevant heating areas. Quality and quantitative analysis could be applied to these data and being diagnosed further to obtain the characteristics of the equipment status in the corresponding parts.

\section{RELATED THEORIES}

\section{A. Thermogram Segmentation}

Thermogram segmentation could be carried out using various segmentation methods. However, this research is concerned with 2 segmentation methods including K-means 
segmentation and Fuzzy C-means segmentation.

\section{1) K-means Segmentation}

K-means is originally developed for iteratively clustering data into $\mathrm{K}$ groups. The classification depends on a specific function used for evaluating whether the terminated criterion is met, and the $\mathrm{K}$ groups result is obtained.

K-means clustering could be accomplished by divide the initial set of data into K groups. For each group, the mean of the group is calculated and represented as the centroid. The centroid is used for measuring the distance among members of the group [5].

K-means algorithm can be described as follows: Step 1: Randomly select the initial $\mathrm{K}$ centroids represented by $C_{k}$. Where $k$ is the number of clusters to be used.

Step 2: Calculate the minimum distance $\mathrm{K}$ between each centroid and the members within the group.

$$
k=\operatorname{argmin}\left\|x_{k}-c_{k}\right\|
$$

where $x_{k}$ represents each individual members within the group, and $c_{k}$ is the centroid of each group $x_{k}$ will be included into group $\mathrm{K}$ if the distance between the centroid of group $\mathrm{K}$ and $x_{k}$ is minimum.

Step 3: Centroids of $\mathrm{K}$ groups will be recalculated by finding mean of each group members as follows:

$$
C_{k}=\frac{1}{\left|q_{i}\right|} \sum_{x_{i} \in q_{i}} x_{i}
$$

where $x_{i}$ is data point, $q_{i}$ is set of data point assignments for each $j^{\text {th }}$ cluster centroid.

Step 4: Calculate total distance $D$, which is the sum of all distances between each centroids and corresponding members within the group. This can be described by the following equation.

$$
D=\sum_{i=1}^{N} \frac{k}{\sum_{j=1}^{k} 1 / d_{i j}}
$$

Notice that $I_{i j}$ is used to represent the membership of group K. If $I_{i j}=1, x_{i}$ is the member of $q_{i}$.

Step 5: Repeat step $2-4$ until the total distance is unchanged:

$$
1-\frac{D^{\text {old }}}{D^{\text {new }}} \leq \propto
$$

For $\propto$ is a very small constant.

\section{2) Fuzzy C-means (FCM)}

Fuzzy C-means (FCM) is a technique for grouping data together which enables one piece of data to have a membership from at least two groups. This strategy created by Dunn in 1973, [6] and adoped to image segmentation by Sulaiman [7] has been regularly utilized as a part of pattern recognition. It depends on minimization of the following objective function :

$$
J_{m}=\sum_{i=1}^{N} \sum_{j=1}^{C} u_{i j}^{m}\left\|x_{i}-c_{j}\right\|^{2}, 1 \leq m<\infty ;
$$

where $m$ is any real number greater than $1, u_{i j}$ is the degree of membership of $x_{i}$ in the cluster $j, x_{i}$ is the $i$ th of $\mathrm{d}$ dimensional measured data, $c_{j}$ is the d-dimension center of the cluster, and $\|*\|$ is any norm expressing the similarity between any measured data and the center.

Fuzzy partitioning is brought out through an iterative optimization of the objective function shown above, with the update of membership $u_{i j}$ and the cluster centers $c_{j}$ by:

$$
\begin{gathered}
c_{j}=\frac{\sum_{i=1}^{N} u_{i j}^{m} \cdot x_{i}}{\sum_{i=1}^{N} u_{i j}^{m}} \\
u_{i j}=\frac{1}{\sum_{k=1}^{C}\left[\left\|x_{i}-c_{j}\right\|\right]^{\frac{2}{m-1}}}
\end{gathered}
$$

This iteration will stop when $\max _{i j}\left\{\left\|u_{i j}^{(k+1)}-u_{i j}^{(k)}\right\|\right\}<$ $\varepsilon$, where $\varepsilon$ is a termination criterion between 0 and 1 , where as $k$ are the iteration steps. This procedure converges to local minimum or saddle point of $J_{m}$.

The algorithm is composed of the following steps:

Step 1: Initialize $U=\left[u_{i j}\right]$ matrix, $U^{(0)}$

Step 2: At k step: calculate the centers vectors $C^{(k)}=\left[c_{j}\right]$ With $U^{(k)}$

$$
c_{j}=\frac{\sum_{i=1}^{N} u_{i j}^{m} \cdot x_{i}}{\sum_{i=1}^{N} u_{i j}^{m}}
$$

Step 3: Update $U^{(k)}, U^{(k+1)}$

$$
u_{i j}=\frac{1}{\sum_{k=1}^{C}\left[\left\|x_{i}-c_{j}\right\|\right]^{\frac{2}{m-1}}}
$$

Step 4: If $\left\|u_{i j}^{(k+1)}-u_{i j}^{(k)}\right\|<\varepsilon \quad$ Then stop. Otherwise return to step 2

\section{B. Geometrical Statistics}

\section{1) Statistics features}

Statistics features can be used to determine the status of thermogram objects. Generally, the most prominent statistics features that could be applied included variance of the image $\left(\sigma^{2}\right)$, mean of image pixel values $(\boldsymbol{\mu})$, and standard deviation $(\boldsymbol{\sigma})$. In this work, mean of image pixel values pays an important role in diagnosis of thermogram. The mean of image pixel values could be calculated as follows:

Mean of image pixel values $(\mu)=\sum \frac{P_{x y}}{x \times y}$

Standard deviation of image

where $\boldsymbol{P}_{\boldsymbol{x} \boldsymbol{y}}$ represents the pixel value at the corresponding position $\mathrm{x}, \mathrm{y}$

\section{2) Geometrical features}

Geometrical features of thermogram objects are concerned with geometric components such as curve, line, surface, point, bulk, and edge. However, this work is focused on the contiguous region of interests, and areas of hot temperature.

\section{TRANSFORMER THERMOGRAM DiAgNOSIS FRAMEWORK}

Fig. 1. Demonstrates the development framework of transformer diagnosis using thermogram. First, the infrared image is acquired in order to be segmented further using Kmeans or Fuzzy Cmeans. The result segmentation will be identified for ROIs and then being analized using 
geometrical statistics and related features. Finally, the status of transformer is carried out by using tolerant score calculation as defined by the following algorithm.

Transformer thermogram diagnosis algorithm:

$$
\begin{aligned}
& K-\operatorname{mean}_{k} / C-\operatorname{means}\left(I_{p_{i}}\right)=\sum_{i=1}^{k} \operatorname{Seg}_{p_{i}} \\
& R O I_{p_{i=1 \rightarrow m}} R O I\left(\operatorname{Seg}_{110 \leq \operatorname{Inten}\left(P_{i}\right) \leq 256}\right)
\end{aligned}
$$

Tolerantscore $_{\mathrm{ROI}}=\frac{\sum_{i=1}^{m}\left[\text { Inten }_{R O I_{i}} \times w_{p}\right]}{n}$

Alert if Score $(p)\left\{\begin{array}{l}\geq 45(P=\text { Bushing }) \rightarrow \text { Alert } \\ \geq 39(P=\text { Tank }) \rightarrow \text { Alert } \\ \geq 28(P=\text { OLTC }) \rightarrow \text { Alert } \\ \geq 24(P=\text { Radiator }) \rightarrow \text { Alert }\end{array}\right.$

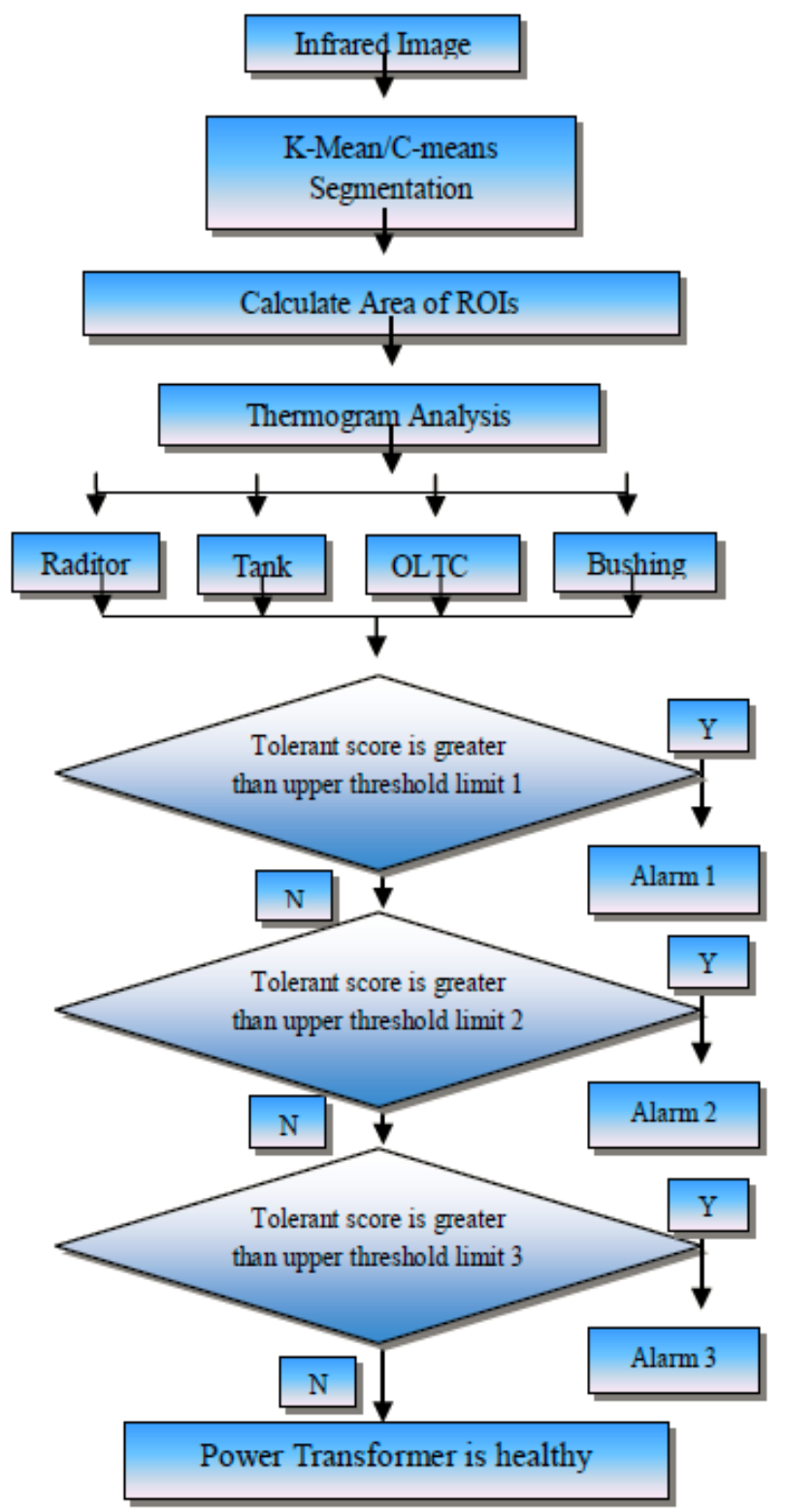

Fig. 1. Transformer thermogram diagnosis framework.

where $I_{p_{i}}$ represents the image of component $p_{i}$ of the transformer, $\operatorname{Seg}_{p_{i}}$ the segmented image of part $i$ of the component $P$ of the transformer, ROI is the Region Of Interest, $i=$ part no. of the transformer component $p, m=$ maximum number of transformer component $p$, Inten $\left(P_{i}\right)$ is the pixel intensity of transformer part $\mathrm{I}$, tolerent score $\mathrm{ROI}_{p}$ is the tolerant score regarding to the ROI of component $p, n=$ number of pixels of the image component, and $w_{p}=$ weigh of the image component $p$.

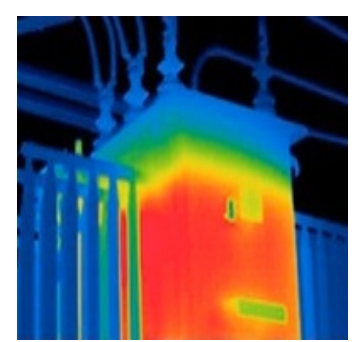

(a) IR Tank

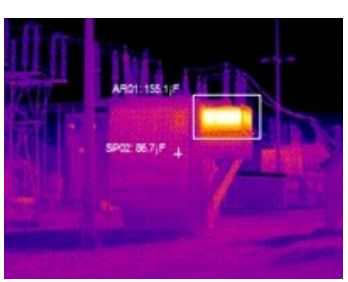

(c) IR OLTC

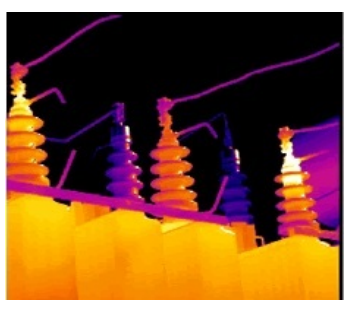

(e) IR Bushing

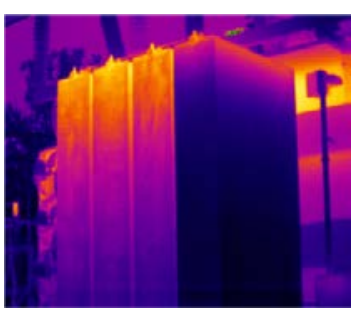

(g) Radiator

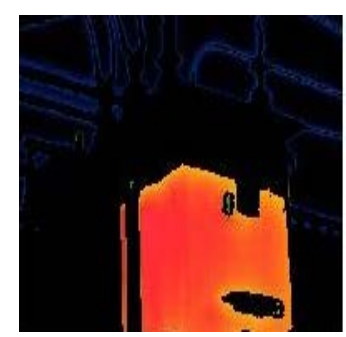

(b) IR Tank Segmented

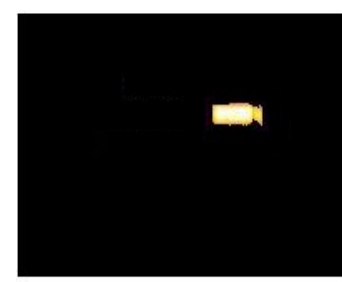

(d) IR OLTC segmented

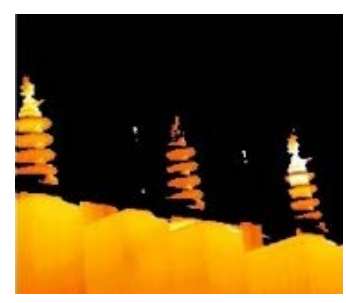

(f) IR Bushing segmented

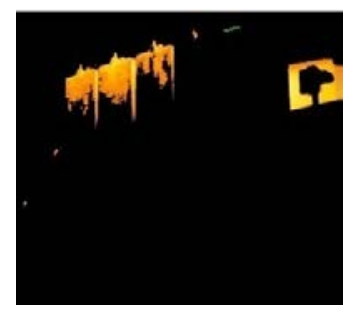

(h) Radiator segmented Fig. 2. Segmentation images obtained by K-mean.

In Fig. 2, (a), (c), (e) and (g) are original thermal images of 4 components of power transformers; (b), (d), (f) and (h) are segmented thermal image using K-means algorithm.

In Fig. 3, (a), (c), (e) and (g) are original thermal images of 4 components of power transformers; (b), (d), (f) and (h) are segmented thermal image using Fuzzy C-means algorithm.

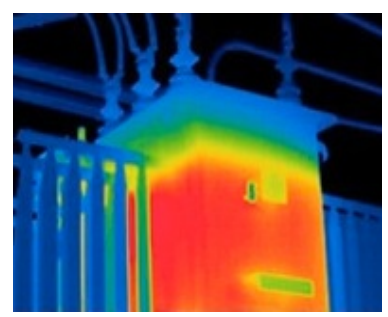

(a) IR Tank

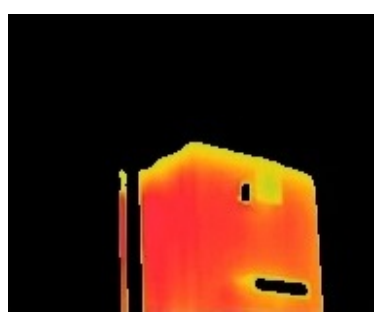

(b) IR Tank Segmented 

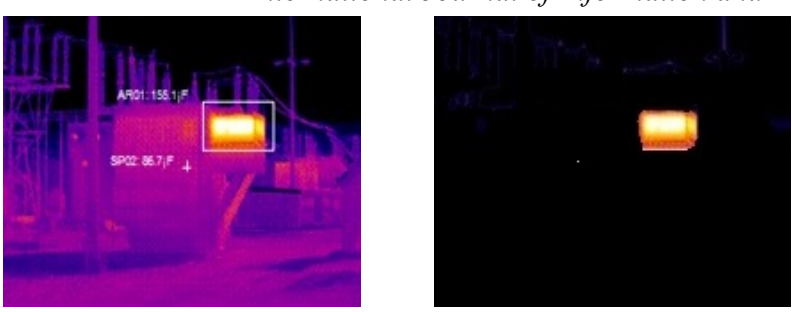

(c) IR OLTC

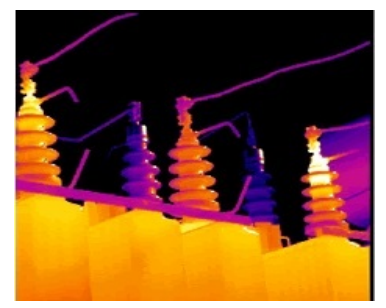

(d) IR OLTC segmented

(e) IR Bushing

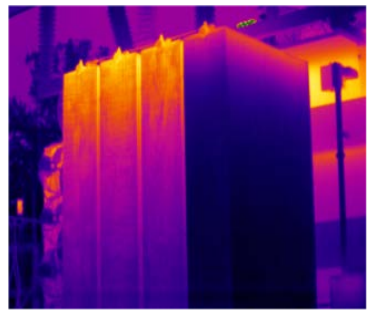

$\begin{array}{ll}\text { (g) Radiator } & \text { (h) Radiator segmented }\end{array}$

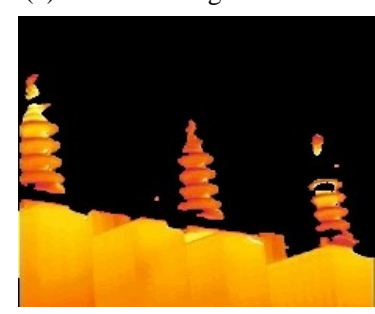

(f) IR Bushing segmented

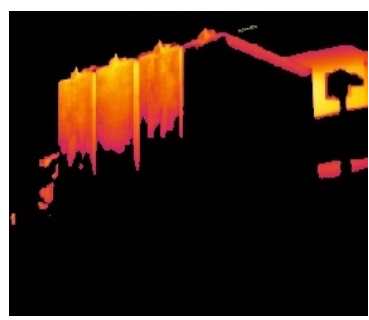

(h) Radiator segmented

\section{EXPERIMENTAL RESULT}

By using K-means and C-means segmentation, the following results had been obtained.

TABLE I: TOLERANT SCORES OF TRANSFORMER COMPONENTS USING K-

\begin{tabular}{ccccc}
\multicolumn{5}{c}{ MEAN SEgMENTATION } \\
\hline \hline $\begin{array}{c}\text { Part of } \\
\text { Transformer }\end{array}$ & $\begin{array}{c}\text { Average } \\
\text { ROI area } \\
\text { (AVG) }\end{array}$ & Wieght & $\begin{array}{c}\text { Tolerant } \\
\text { Score } \\
\text { (W) }\end{array}$ & AVG ROI \\
\hline Bushing & 210.22 & 0.3 & 63.06 & 82.43 \\
Tank & 177.36 & 0.3 & 53.2 & 69.55 \\
OLTC & 195.26 & 0.2 & 58.57 & 76.57 \\
Radiator & 167.78 & 0.2 & 33.55 & 65.79 \\
\hline \hline
\end{tabular}

TABLE II: TOLERANT SCORES OF TRANSFORMER COMPONENTS USING FUZZY C-MEANS SEGMENTATION (FCM)

\begin{tabular}{ccccc}
\hline \hline $\begin{array}{c}\text { Part of } \\
\text { Transformer }\end{array}$ & $\begin{array}{c}\text { Average } \\
\text { ROI area } \\
\text { (AVG) }\end{array}$ & Wieght & $\begin{array}{c}\text { Tolerant } \\
\text { Score } \\
\text { (W) }\end{array}$ & AVG ROI \\
\hline Bushing & 166.38 & 0.3 & 49.91 & 64.99 \\
Tank & 138.96 & 0.3 & 41.68 & 54.28 \\
OLTC & 130.83 & 0.2 & 26.16 & 51.1 \\
Radiator & 132.38 & 0.2 & 26.47 & 51.71 \\
\hline \hline
\end{tabular}

The values of Weight parameter could be varied depending on different components of the transformer. Empirically, the components that are risky to be damaged often should have more weight than that of less risky components. Accordingly, Bushing and Tank were likely to be more riskly of being damaged than Tap changer and Radiator as revealed by the weight of 0.3 , and 0.2 , respectively.

Regarding Table1 and 2, the values of AVG ROI area , score and \% of AVG ROI obtained from K-means and Fuzzy C-means Segmentation were significantly different. In this context, K-means was preferable due to its capability to identify the unique range of tolerant scores significantly.

\section{CONCLUSION}

The proposed power transformers diagnosis framework using infrared thermograms analysis revealed the tolerant scores of transformer components. Therefore, these scores were ralated to simple conclusion for the possiblilities that some problems could occur to the Tank due to low oil level, which led to the exceeding heat. The increasing heat of OLTC was caused from the contact problem. Bushing temperature was increasing due to loose joints. Moreover, Radiator overheat could be affected by high load.

Equipment maintenance is very important, which is in accordance with Electricity Du Laos's goal to preserve stability and maintenance cost in power stations. Transformer diagnosis using thermogram is considered to be a good option to detect exceeding heat in transformer components, and to help planing in advance for dealing with possible damages to different components of transformer effectively.

\section{FUTURE WORK}

The proposed framework could be extended to function with the estimation of the MTBF (Mean Time Between Failures) of the equipment. This is challenging to find MTBF of the equipment using thermogram processing, which could be more economy and less time consuming process comparing to the conventional methods. The tolerant scores should be transformed in order to be in consistent with MTBF for helping with maintenance planning in the future.

\section{REFERENCE}

[1] Z. A. Jaffery and A. K. Dubey, "Design of early fault detection technique for electrical assets using Infrared thermograms,” Journal of Infrared Physics \& Technology, vol. 63, pp. 753-759, 2014.

[2] M. S. Jadin and S. Taib, "Recent progress in diagnosing the reliability of electrical equipment by using infrared thermography," Journal of Infrared Physics \& Technology, vol. 55, pp. 236-245, 2012.

[3] Y. C. Chou and L. Yao, "Automatic diagnosis of electrical equipment using infrared thermography," in Proc. Int. Conf. on Soft Computing and Pattern Recognition, IEEE Computer Society, 2009, pp. 155-160.

[4] T. Dutta, J. Sil, and P. Chottopadhyay, "Condition monitoring of electrical equipment using thermal image processing," in Proc. IEEE First International Conference on Control, 2016, pp. 978-1-47991769.

[5] R. Sammouda, H. Aboalsamh, and F. Saeed, "Comparison between K mean and fuzzy C-Mean methods for segmentation of near infrared fluorescent image for diagnosing prostate cancer,” in Proc. IEEE Computer Vision and Image Analysis Applications (ICCVIA), 2015, pp. 978-1-4799-7186.

[6] J. C. Dunn, "A fuzzy relative of the ISODATA process and its use in detecting compact well-separated clusters," Journal of Cybernetics, pp.32-57, 2008.

[7] S. N. Sulaiman and N. A. M. Isa, "Denoising-based clustering algorithms for segmentation of low level 
salt-and-peppernoise-corrupted images," in Proc. IEEE Trans. Consum. Electr., 2010, vol. 56, pp. 2702-2710.

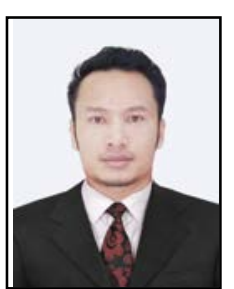

Phoutsavanh Chandavong received the bachelor's degree in electrical engineering from Danang University of Technology in 2010.

After that he had been working at Seatech Company, Danang, Vietnam , for six months before joining the Department of Technical Electric, Electricite Du Laos, Saravanh Brand, Lao PDR , in 2010. Since 2012, he has been working at Sounthern Transmission System Office, Electricite Du Lao and being appointed as a head of Nathone Substation,

Saravanh Province.

He is currently a graduate student in engineering technology program, Ubon Ratchathani Rajabhat University, Thailand.

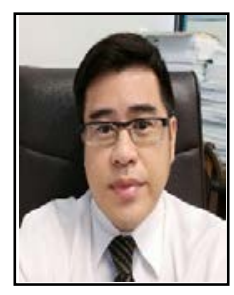

Danu Wiroteurairuang received his master degree in computer science from Old Dominion University, USA in 2003.

He finished his doctor of engineering in electrical engineering from Mahanakorn University of Technology in 2015.

$\mathrm{He}$ is an assistant professor and currently a full time lecturer at Engineering Technology Program, Faculty of Industrial Technology, Ubon Ratchathani Rajabhat University, Thailand. His recent research interests include image processing, pattern recognition, and artificial intelligent. 